\title{
AIAA 2002-4411 \\ Aerodynamic Database \\ Development for Mars Smart \\ Lander Vehicle Configurations
}

Glenn J. Bobskill and Paresh C. Parikh

NASA Langley Research Center

Hampton VA

Ramadas K. Prabhu

Lockheed Martin Engineering and Sciences Corporation

Hampton VA

Erik D.Tyler

Swales Aerospace

Hampton VA

\section{AIAA Atmospheric Flight Mechanics Conference \& Exhibit \\ August 5-8, 2002 \\ Monterev. CA}

For permisclas to copy or to republish, contaxt the copyrtight owner nomed an the first pape-

For AlAA-held copjripht, wite to AMA Permissiens Department,

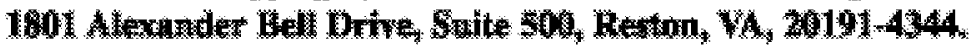




\title{
AERODYNAMIC DATABASE DEVELOPMENT FOR MARS SMART LANDER VEHICLE CONFIGURATIONS
}

\author{
Glenn J. Bobskill ${ }^{*}$ and Paresh C. Parikh ${ }^{\dagger}$ \\ NASA Langley Research Center, Hampton, VA 23681 \\ Ramadas K. Prabhu ${ }^{\ddagger}$ \\ Lockheed Martin Engineering and Sciences Corporation, Hampton, VA 23681 \\ Erik D. Tyler ${ }^{\hat{\beta}}$ \\ Swales Aerospace, Hampton, VA 23681
}

\begin{abstract}
An aerodynamic database has been generated for the Mars Smart Lander Shelf-All configuration using computational fluid dynamics (CFD) simulations. Three different CFD codes, USM3D and FELISA, based on unstructured grid technology and LAURA, an established and validated structured CFD code, were used. As part of this database development, the results for the Mars continuum were validated with experimental data and comparisons made where applicable. The validation of USM3D and LAURA with the Unitary experimental data, the use of intermediate LAURA check analyses, as well as the validation of FELISA with the Mach $6 \mathrm{CF}_{4}$ experimental data provided a higher confidence in the ability for CFD to provide aerodynamic data in order to determine the static trim characteristics for longitudinal stability. The analyses of the noncontinuum regime showed the existence of multiple trim angles of attack that can be unstable or stable trim points. This information is needed to design guidance controller throughout the trajectory.
\end{abstract}

* Aerospace Engineer, Vehicle Analysis Branch, Aerospace Systems, Concepts, and Analysis Competency, Member AIAA

$\uparrow$ Senior Aerospace Engineer, Configuration Aerodynamics Branch, Aerodynamics, Aerothermodynamics, and Acoustics Competency,

Associate Fellow AIAA

¥ Senior Aerospace Engineer

$\S$ Aerospace Engineer

Copyright 2002 by the American Institute of Aeronautics and Astronautics, Inc. No copyright is asserted in the United States under Title 17, U.S. Code. The U.S. Government has a royalty-free license to exercise all rights under the copyright claimed herein for Governmental Purposes. All other rights are reserved by the copyright owner

\section{Nomenclature}

$A$

$B C$

$C A$

$C D$

$C G$

$C N$

$\mathrm{Cm}$

Kn

$L D$

$m$

$M$

$\alpha$

$\gamma$

$\mathrm{Cm} m_{\alpha}$

Subscripts:

trim
Reference area $\left(\mathrm{m}^{2}\right)$

Ballistic coefficient $(\mathrm{m} / \mathrm{CD} * \mathrm{~A})$

Axial force coefficient

Drag coefficient

Center of gravity

Normal force coefficient

Pitching moment coefficient

Knudsen number

Lift to drag ratio

Vehicle mass $(\mathrm{kg})$

Mach number

Angle of attack

Ratio of specific heats

Static stability derivative

trim condition 


\section{Introduction}

The exploration of Mars has been driven by the need to understand its planetary evolution and thereby recognize its past, present, or future potential to support life. The discovery of Martian life would require the ability to understand or characterize Mars from its geology, climate, biology, and other natural processes. This requirement ultimately defines a set of overall science objectives/payloads along with a probable set of planetary surface locations. In addition, the accuracy of targeting a specific surface location and achieving a successful precision landing are also essential requirements for fulfilling these science objectives.

The entry, descent, and landing (EDL) phases for a Mars mission can be categorized based on $1^{\text {st }}, 2^{\text {nd }}$, and $3^{\text {rd }}$ generation type systems. ${ }^{1}$ These categories describe the development of landing ellipses based on large scale $(100 \mathrm{~s}$ of $\mathrm{km})$, precision $(<10 \mathrm{~km})$, and pinpoint distance accuracies from the desired landing site. The $1^{\text {st }}$ generation EDL system utilizes a ballistic entry. The $2^{\text {nd }}$ generation EDL system (Smart) employs significant improvements in guidance and navigation methods ${ }^{2-3}$ enabling precision entry and utilizes a "Smart" or local surface hazard avoidance and hazard tolerance system in order to provide a safer landing environment. The $2^{\text {nd }}$ generation EDL system also requires the development of a higher fidelity trajectory simulation. The objectives are for the vehicle to land within $10 \mathrm{~km}$ of the intended touchdown site and landing safely $99.7 \%$ of the time.

The trajectory simulation for the Mars Smart Lander (MSL) configuration series was performed using the 3-degree of freedom (DOF) version of the POST ${ }^{4}$ (Program to Optimize Simulated Trajectories). The POST program integrates the equations of motion and includes a set of specific constraints necessary to define the overall planetary, mission, and vehicle parameters. These models include specific science-related information of the planet Mars such as planet definition, gravity, and atmosphere while other models provide specific information related to the vehicle configuration and the vehicle characteristics of motion. Examples of these models include control system emulation, guidance algorithms, navigation system, mass properties, and aerodynamics. The aerodynamics model is a database that provides aerodynamic force and moment data of a specific vehicle configuration for both the entry and descent segments of the trajectory. The entry phase is defined as the region between the atmospheric interface and the supersonic parachute deployment point while the descent phase is defined from that point to landing.
The development of an entry aerodynamic database requires specific knowledge of the geometry and orientation of the vehicle in addition to the freestream quantities along a given trajectory. In accordance with the Mars Smart Lander payload and surface location requirements, a set of preliminary configurations were developed that would have favorable trim characteristics along proposed nominal and dispersed trajectories. These vehicle configurations (Figure 1) are the axisymmetric Baseline, Canted-All, Shelf-All, and a derivative of the Shelf-All tested in the Ames Ballistic range facility. All configurations have the same $70^{\circ}$ forebody cone shape with a bi-conic backshell. The Shelf-All model was designed with a blended control tab that is tangent to the forebody surface while the Shelf-2 model has a non-blended control tab while also being tangent to the forebody surface. The Canted-All model has a similar Shelf-All control tab but is canted $10^{\circ}$ towards the vehicle nose.

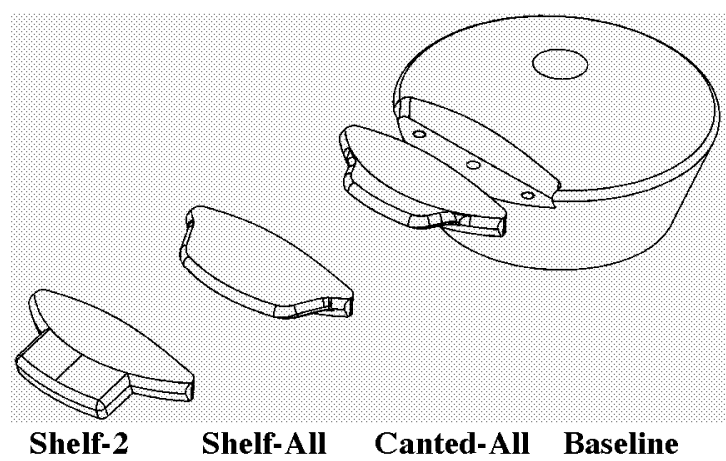

Figure 1. Mars Smart Lander Configurations

The objectives of this paper are to provide information on the development of an entry aerodynamic database, to provide specific validation of the CFD analysis programs with experimental data, and show the resulting flight trim characteristics for a Mars Smart Lander configuration. The subsequent sections of this paper will describe the analysis codes used, CFD validation, and the creation and results of a flight database.

\section{Code Descriptions}

\section{USM3D}

USM3Dns ${ }^{5}$ is a three dimensional, tetrahedral, cell-centered, finite volume Euler and Navier-Stokes flow solver for unstructured meshes. Inviscid flux quantities are computed across each cell face using Roe's flux-difference splitting (FDS) ${ }^{6}$. Spatial discreti- 
zation is accomplished using an analytical reconstruction process for computing solution gradients within tetrahedral cells. The solution is advanced in time to a steady state condition by an implicit backward Euler time-stepping scheme. Flow turbulence is modeled by the Spalart-Allmaras one-equation model, which is optionally coupled with a wall-function formulation to reduce solution stiffness and the number of cells in the sub layer of the boundary layer. USM3Dns runs on massively parallel computers and clusters of personal computers (PC's). Although a single processor version is available for a variety of computing platforms, the parallel version ${ }^{7}$ is the code of choice because it enables rapid turnaround for large problems.

Although USM3Dns is widely in use by aerospace practitioners for several years, the latest Navier-Stokes version was never validated for the Mach number range and shape (especially the blunt fore body) of interest in the present study. In addition, the available version was so far used with perfect gas assumptions, while the aerodynamic database required solutions at Mars atmospheric conditions involving a different gas constant. For these reasons, the flow solver had to be modified before application. This section briefly describes the changes made to the code. The following changes were incorporated to the existing parallel version of USM3Dns:

1. A new flow initialization procedure was introduced whereby for the cases were the freestream Mach number exceeded 1.2; the solution was initialized to a lower, usually subsonic, value. This strategy helped the flow solution convergence to steady state, especially in the aft, low-Mach number region of the configurations.

2. A special flux-splitting scheme based on the AUSM $^{8}$ (Advection Upstream Splitting Method) scheme was incorporated to overcome the well-known "Carbuncle phenomenon" usually associated with the application of the Roe scheme to a case with strong shock waves.

3. In order to simulate the Mars atmospheric conditions, the perfect gas assumptions used thus far was modified to allow specification of an effective $\gamma$ for each freestream Mach number, along with coefficients for Sutherland's for viscosity.

\section{LAURA}

The Langley Aerothermodynamic Upwind Relaxation Algorithm (LAURA) is a three dimensional $\mathrm{Na}$ -
vier-Stokes solver developed by Gnoffo ${ }^{9-11}$ for structured grids. LAURA is a finite volume formulation of the integral form of the Navier-Stokes equations. A second order-accurate, symmetric total-variationdiminishing (TVD) scheme ${ }^{12}$ is used in conjunction with upwind differencing of the discretized equations. At each cell face, Roe's averaging defines the flowfield variables based on values from the adjacent cells. The unsteady governing equations are driven to a steady state solution through an implicit time relaxation procedure. During the relaxation process, the grid is periodically adapted in the body-normal direction so that the grid can be tailored to the emerging solution. This elliptic flow solver has the capability of solving chemical non-equilibrium flow through the specification of chemical species and reactions unique to a specific atmosphere as well as the ability to model perfect gas and equilibrium air.

\section{FELISA}

The FELISA ${ }^{13-14}$ (Finite Element Langley Imperial College Swansea Ames) software system consists of unstructured surface and volume grid generation with an inviscid flow solver and unstructured post processing utilities. The flow algorithm was developed through the application of the Galerkin finite element method in space to obtain a coupled set of ordinary differential equations in time. The steady state solution of this equation set is achieved by advancing the system using an explicit Runge-Kutta type marching scheme. The hypersonic flow solver has options for perfect gas, equilibrium air, $\mathrm{CF}_{4}, \mathrm{CO}_{2}$, and Mars equilibrium gases. In addition, the hypersonic flow solver has the capability of solving chemical non-equilibrium flow and real gas (chemical and thermal non-equilibrium) flow.

\section{Aerodynamic Database}

The aerodynamic database is provided as a FORTRAN routine containing specific vehicle force and moment data as a function of vehicle orientation relative to the flow and flowfield parameters to the POST trajectory simulation. Within this routine, the vehicle trajectory is partitioned into three atmospheric regimes that are defined based on the similarity parameter of Knudsen number. The Knudsen number $(\mathrm{Kn})$ is defined as the mean free path (distance between molecular collisions) divided by the vehicle reference length (typically the aeroshell diameter). These atmospheric regimes are depicted in Figure 2. 


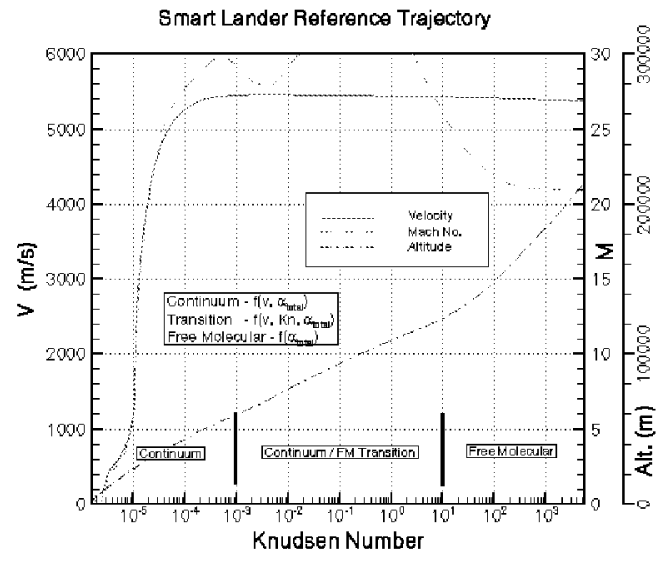

Figure 2. Mars Smart Lander Reference Trajectory

The aerodynamic database is comprised of continuum, transitional, and free molecular ${ }^{15-16}$ regimes that are accessed based on the calculated local Knudsen number at any entry trajectory point. Each section consists of axial force, normal force, and pitching moment data that was created from specific CFD analysis points as well as experimental data ${ }^{17}$ that have been corrected for testing environment differences. Since the trajectory simulation is normally run under nominal and offnominal conditions during prescribed Monte Carlo analyses, the aerodynamic database has to be sufficiently populated at several freestream Mach numbers and angles of attack. To determine values within or outside the Mach number and angle of attack ranges, a bi-parabolic/linear interpolation scheme is applied within the range of the database.

Within the continuum regime, three different CFD analysis programs were used to obtain direct flight aerodynamic data or to provide necessary corrections to the Unitary experimental data for flight condition applicability while the non-continuum flight data was determined using the program as mentioned in References 15 and 16. The USM3Dns code was used to perform the Unitary data corrections from the predicted low supersonic parachute deploy boundary to the upper supersonic limit of the Unitary facility. The FELISA results provided the aerodynamic flight results from a mid-hypersonic to upper hypersonic trajectory point that corresponded to the approximate atmospheric continuum limit. Lastly, the LAURA results provided intermediate analysis comparisons that overlap the above trajectory ranges. The proposed continuum flight cases are shown in Table 1.
The selections and application of these analysis programs were based on several considerations. The first consideration was to develop a methodology that could allow for rapid turnaround of assessing trends for proposed vehicle configurations by taking advantage of grid generation methods and flowfield modeling. Another consideration was the flight regime capability of each program. The final consideration was to ensure confidence and continuity within the solution matrix by using the LAURA code to validate intermediate analysis points with the USM3D and FELISA analysis programs since LAURA has been previously validated with previous Mars missions ${ }^{18}$.

Table 1. Proposed Flight Analysis Cases for MSL Reference Trajectory.

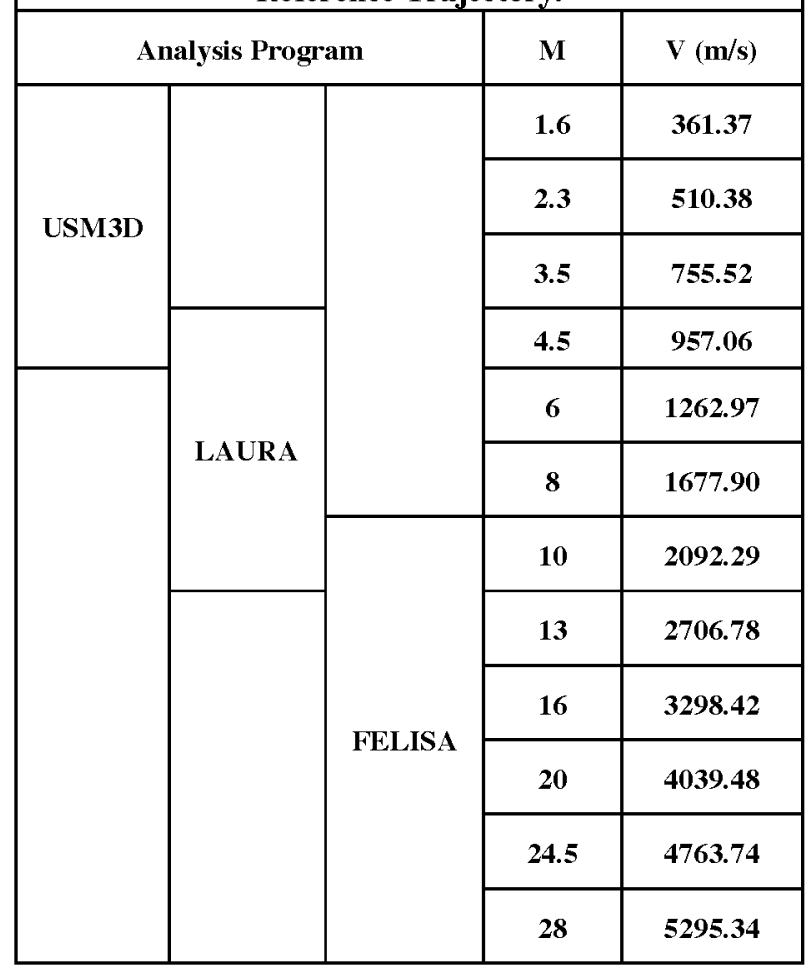

\section{Experimental Data}

Two experimental facilities were used to acquire aerodynamic data for CFD validation of the aforementioned analysis programs. The first facility, the Langley Unitary Plan Wind Tunnel, is a closed-circuit, continuous flow, variable density supersonic tunnel that was used to obtain 6-DOF static aerodynamic data for 4 proposed Mars Lander configurations over a Mach number range of 2.3 to 4.5 . The complete details of the models, facility calibration, instrumentation, and data acquisition are presented in Reference 17. The second 
facility, the 20-inch Mach $6 \mathrm{CF}_{4}$ Tunnel, was used to obtain aerodynamic data for the Mars Surveyor configuration. The details of this work are presented in Reference 19.

\section{Results and Discussion}

\section{USM3Dns Validation}

The USM3D code was required to validate its results with Unitary tunnel experimental data in order to provide confidence in correcting the Unitary Wind tunnel data for the geometric differences between wind tunnel and flight configuration and gas composition of the Mars atmosphere. Along with the previously described changes to USM3D, an unstructured mesh was constructed for the Baseline and Shelf-All tunnel configurations for a series of Mach numbers between 2.3 and 4.5 , each for a range of angles-of-attack values. The grid for the full Navier-Stokes solution varied in size typically from 1.4 million tetrahedral cells for the windtunnel cases to 2.6 million cells for the flight configurations. All the calculations were run for a Reynolds number of 1.0 million based on the maximum diameter of the configuration ( 6 inches for the wind tunnel models and 4.05 meters for the flight cases). The CFD cases were run using up to 60 processors on an SGI Origin 2000 computer. For all the computations reported here, a full viscous formulation was used. Some examples of the unstructured surface topology are depicted in Figures 3 and 4 .

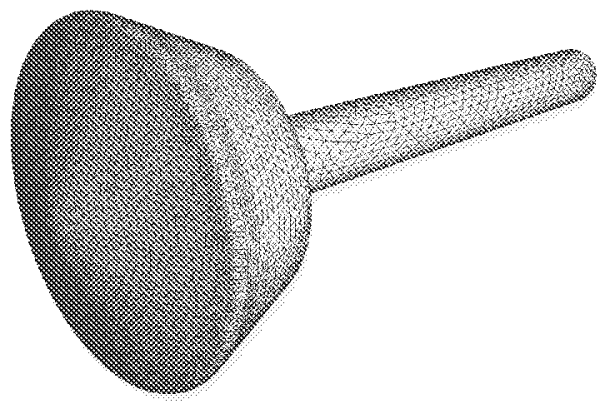

Figure 3. Unstructured Surface Grid for the Unitary Baseline Model Configuration.

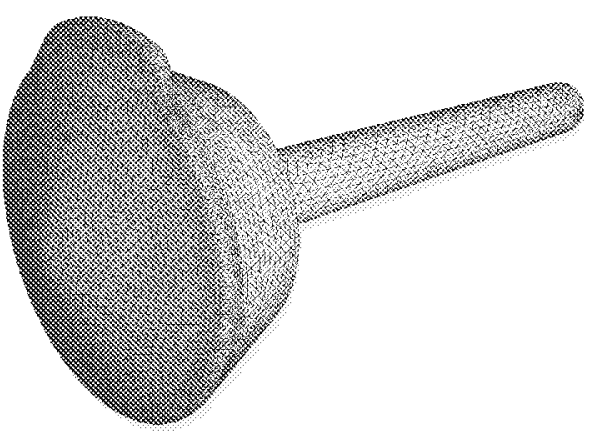

Figure 4. Unstructured Surface Grid for the Unitary Shelf-All Model Configuration.

Figure 5 for the Baseline configuration shows the USM3D/Unitary comparisons for freestream Mach numbers of 2.3 and 3.5. The axial and normal forces along with the pitching moment (about the nose) are shown. The CFD results for the tunnel conditions $(\gamma=1.4)$ compare well against the Unitary wind tunnel data, attesting to the accuracy of the modified solver. The detailed comparisons show a better overall agreement at the Mach 3.5 condition then at the lower Mach 2.3 condition while the Mach 2.3 condition also depicting smaller differences in axial and normal force at 0 and 20 degrees than the intermediate angle of attack range.
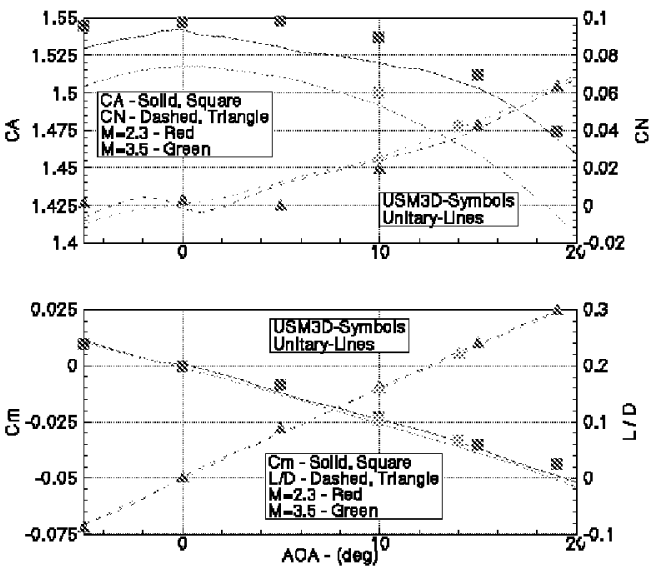

Figure 5. USM3D/Unitary Data Comparisons for the Baseline Configuration.

However, the USM3D analysis does predict a change in normal force slope near zero degrees angle of attack 
which is a known trend for axisymmetric blunt body configurations at low supersonic speeds.

Figure 6 shows the comparisons for the Shelf-All configuration to the Unitary experimental data at freestream Mach numbers of 2.3, 3.5 and 4.5. As with the previous comparisons shown for the Baseline configuration, the overall agreement for Shelf-All configuration is very good. These comparisons show better agreement in axial and normal force at lower Mach numbers than shown for the Baseline model while the pitching moment and L/D distribution shows excellent agreement throughout the Mach number and angle of attack range.
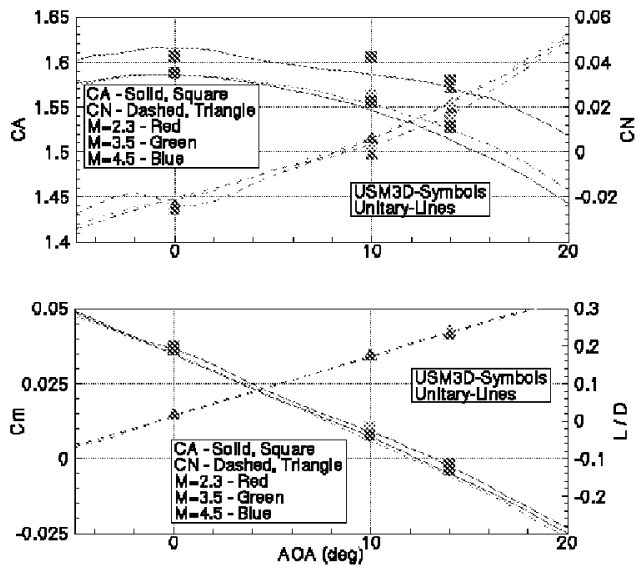

Figure 6. USM3D/Unitary Data Comparisons for the Shelf-All Configuration.

Figure 7 shows the comparisons for the Shelf-All configuration to the Unitary experimental data under a sideslip condition of 4 degrees. As with the previous comparisons shown for the Baseline configuration, the overall agreement for Shelf-All configuration is very good. This particular case (6-DOF) was chosen in order to increase the level of validation and thereby illustrate any deficiencies due to a geometry change and under sideslip conditions. These comparisons show a better agreement in axial force and a similar difference in normal force as compared to the non-sideslip freestream condition. Likewise, the comparisons to the pitching moment and $\mathrm{L} / \mathrm{D}$ are excellent. Further assessment of CFD validation would require some solutions at lower angles of attack, but the overall assessment is extremely good. It should also be noted that this sideslip case was not used in the creation of the flight database that is presented in this study.
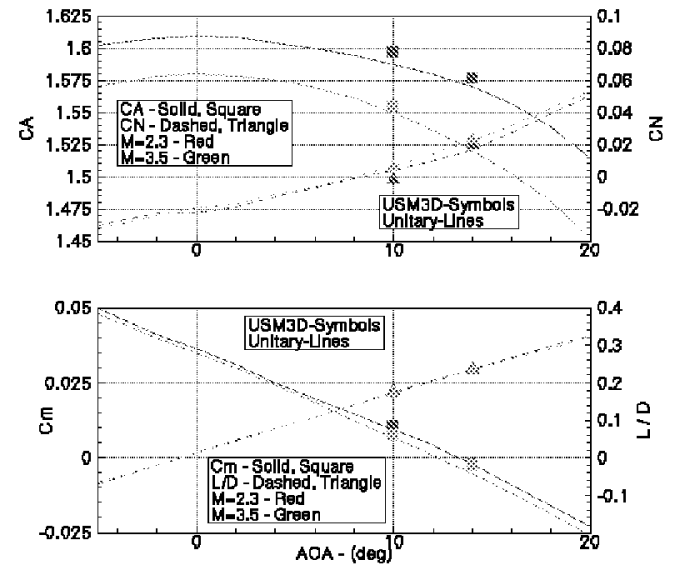

Figure 7. USM3D/Unitary Data Sideslip Comparisons for the Shelf-All Configuration $\left(\beta=4^{\circ}\right)$.

Figure 8 shows the comparisons for the Canted-All configuration to the Unitary experimental data at freestream Mach numbers of 2.3 and 3.5. As with the previous comparisons shown for the Baseline configuration, the overall agreement for Canted-All configuration is very good. These comparisons show better agreement throughout the Mach number and angle of attack range for all force, moment and L/D quantities
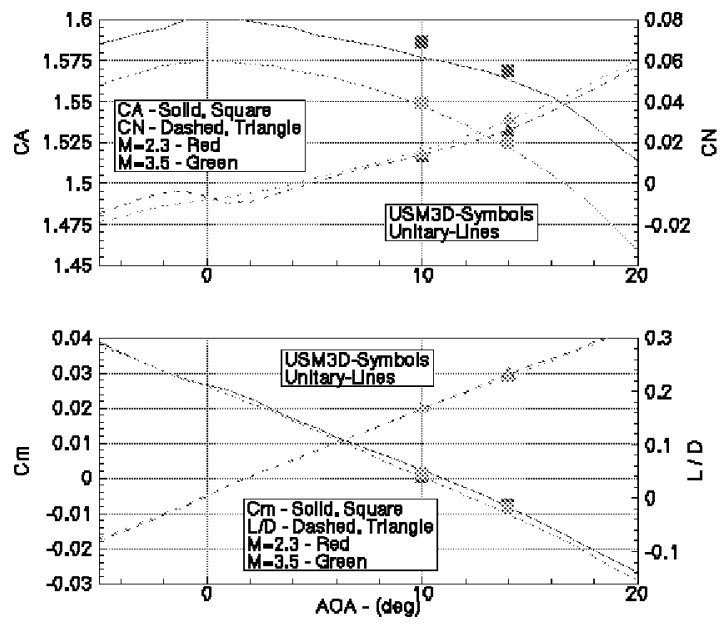

Figure 8. USM3D/Unitary Data Comparisons for the Canted-All Configuration.

than those shown for the Baseline configuration. However, additional solutions at lower angles of attack would have to be made in order to obtain a more complete assessment of CFD validation. 


\section{LAURA Validation}

Although the LAURA analysis program has been previously validated with Mars Pathfinder hypersonic flight data ${ }^{18}$, an attempt was made to provide additional validation with the Unitary experimental data for a single Mars Smart Lander configuration at supersonic speeds and to further validate USM3D. Figure 9 shows the LAURA structured grid topology for the Unitary Baseline configuration. This structured grid is comprised of 16 computational blocks with an approximate total of 450,000 cell centers. In addition, this structured grid was adapted in the direction perpendicular to the body surface as a function of the flowfield temperature and grid distribution values.

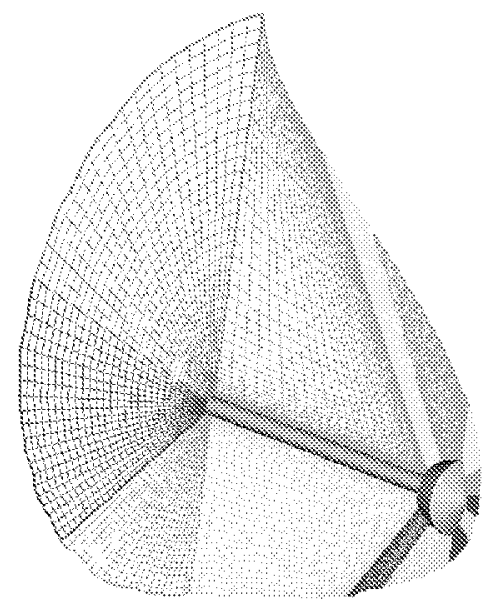

Figure 9. Structured Grid for the Unitary Baseline Model Configuration

Figure 10 depicts the LAURA Mach number contours for the Baseline Unitary tunnel configuration for freestream Mach number of 4.5 and an angle of attack of 10 degrees with no sideslip angle. This solution imposed perfect gas air $(\gamma=1.4)$ at $M=4.5$ tunnel conditions with a laminar, no-slip adiabatic wall boundary condition. Figure 10 shows along the vehicle symmetry and outflow planes the general flowfield characteristics of an axisymmetric blunt body with dominant subsonic regions within the shock layer and vehicle base.

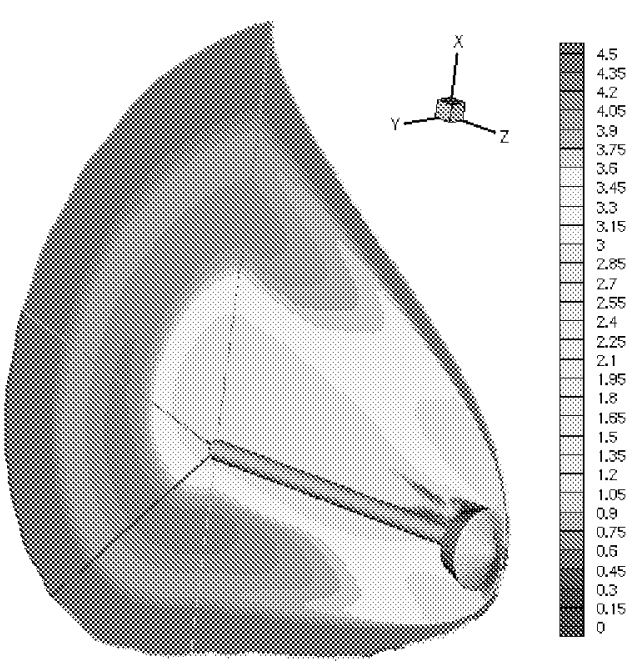

Figure 10. Mach Number Contours for the Unitary Baseline Configuration - $\left(M=4.5, \alpha=10^{\circ}\right)$

Figure 11 depicts the LAURA/Unitary comparisons for the Baseline Unitary tunnel configuration for freestream Mach numbers of 2.3 and 4.5. These comparisons overall show good agreement to the Unitary data for all quantities. The axial force comparisons show better agreement at the higher angles of attack for both Mach numbers while the normal force comparison shows better agreement over the entire angle of attack range at Mach 4.5 while the 10 degree case for Mach 2.3 shows a larger difference as compared to the USM3D/Unitary comparisons in Figure 5.
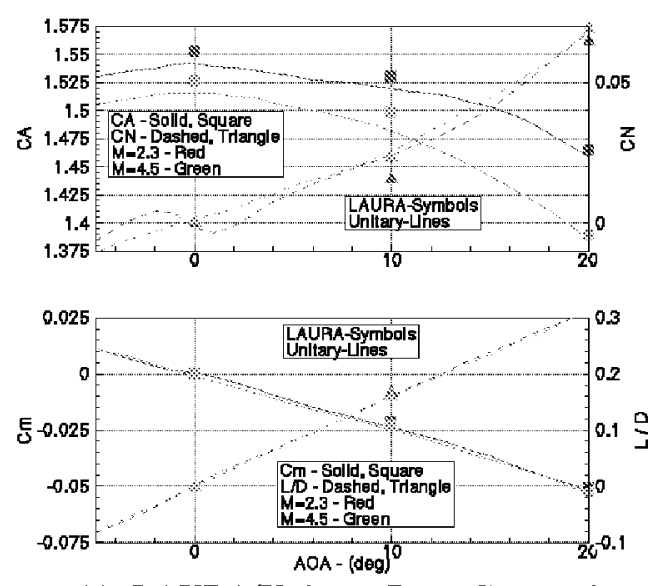

Figure 11. LAURA/Unitary Data Comparisons for the Baseline Configuration. 


\section{FELISA Validation}

The FELISA validation work was centered about an earlier Mars 2001 Surveyor configuration that was tested in the 20-inch Mach $6 \mathrm{CF}_{4}$ Tunnel. The Mars Surveyor configuration (Figure 12) was similar to Mars Smart Lander configuration, but differed in the control flap geometry and location. The Mars Surveyor flap surface area was at least 50 percent smaller and the flap orientation was 90 degrees perpendicular from the axis of rotation and located at aft of the maximum diameter. The number assigned to each control flap indicated the area ratio of the flap where the larger number has a greater area ratio.

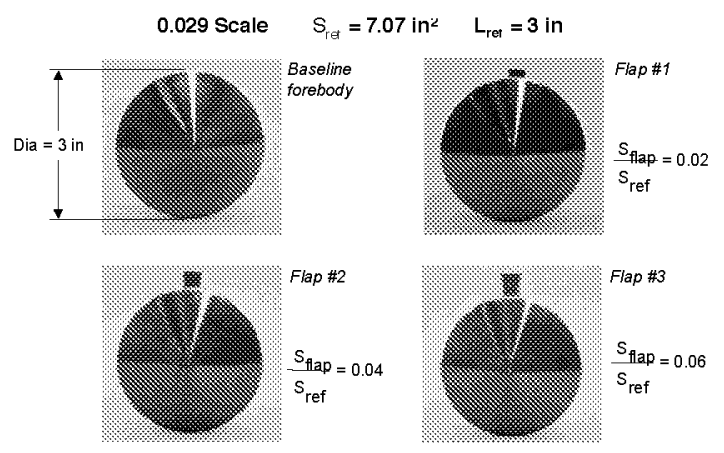

Figure 12. Mars 2001 Surveyor Wind Tunnel Model

Figures 13 and 14 depict the pitching moment coefficient and L/D ratio for the Mars Surveyor configuration for Flap 1. The FELISA $\mathrm{CF}_{4}$ results showed very good agreement to the experimental $\mathrm{CF}_{4}$ data comparisons at $0,5,11$, and 16 degrees angle of attack. In conjunction with a planned test in the 20-inch Mach 6 facility, a FELISA analysis was performed using a $\mathrm{CO}_{2}$ test gas as a freestream condition. The FELISA results predicted that the force and moment contributions to the $\mathrm{L} / \mathrm{D}$ ratio at 0 degrees angle of attack would be insensitive to the $\mathrm{CO}_{2}$ test gas. However, the need for additional results at different angles of attack would be necessary in order to determine a more generalized trend in $\mathrm{CO} 2$ insensitivity.

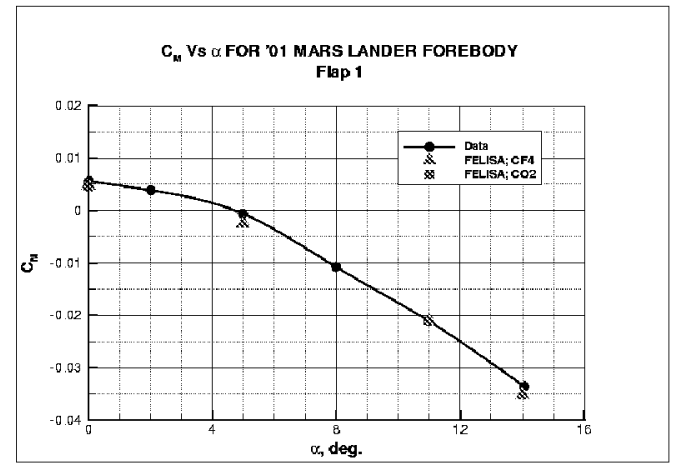

Figure 13. FELISA/CF 4 Pitching Moment Comparisons for the Mars Surveyor Configuration.

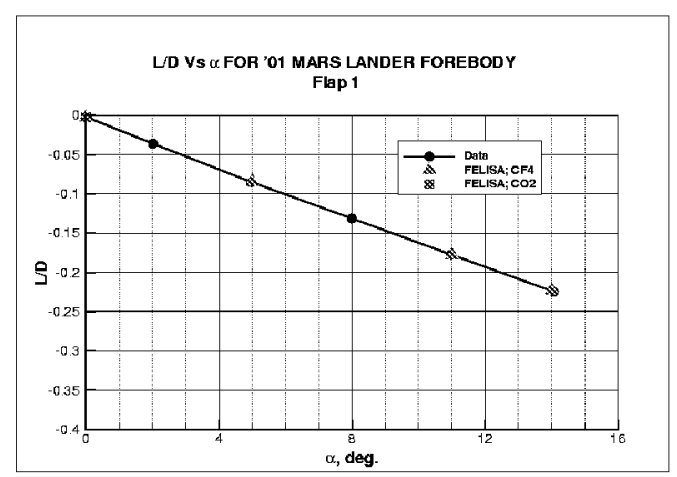

Figure 14. FELISA/CF $\mathrm{L} / \mathrm{D}$ Comparisons for the Mars Surveyor Configuration.

Figures 15 and 16 depict the pitching moment coefficient and L/D ratio for the Mars Surveyor configuration with Flap 3. The FELISA $\mathrm{CF}_{4}$ results shows very good agreement to the experimental $\mathrm{CF}_{4}$ data comparisons at $0,5,11$, and 16 degrees angle of attack. The larger Flap 3 configuration does show a slightly larger deviation in pitching moment at 0 and 5 degrees as compared to the Flap1, but overall agreement is still very good. These differences could be attributed to viscous contributions (not being modeled in FELISA) induced by the larger flap or an indication of a required increase in control flap grid resolution. 


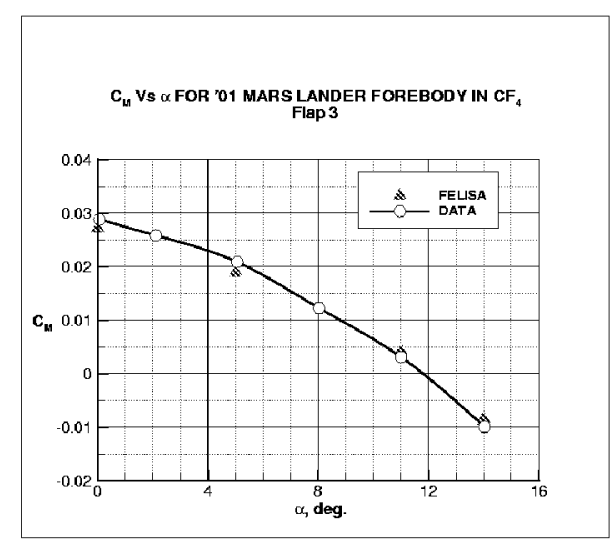

Figure 15. FELISA/CF 4 Pitching Moment Comparisons for the Mars Surveyor Configuration.

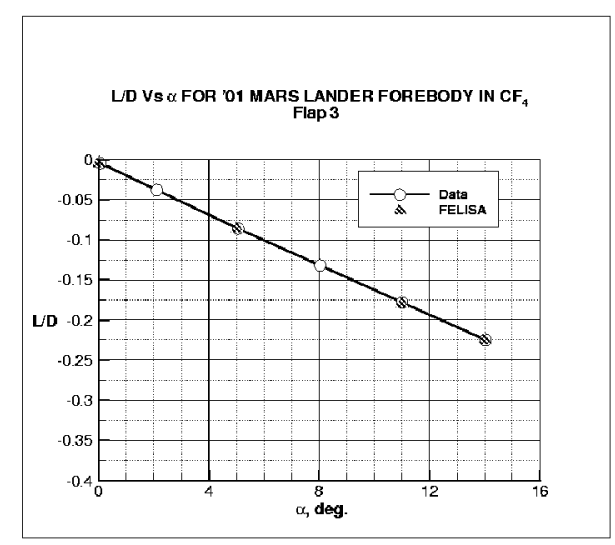

Figure 16. FELISA/CF $\mathrm{L} / \mathrm{D}$ Comparisons for the Mars Surveyor Configuration.

\section{Flight Results - (Shelf-All Continuum Database)}

For the continuum regime, an aerodynamic database was created for the Mars Smart Lander "Shelf-All" configuration using the USM3Dns correction analyses for the Unitary experimental data $(\mathrm{M}=2.3-4.5)$, the LAURA intermediate analysis check points at Mach 4.5 and 10, and the FELISA analyses at Mach 6, 8, 10, 24, and 28. Figures 17 and 18 show the continuum trim characteristics of the Shelf-All configuration based on the axial CG locations of ( $\mathrm{x} /$ Diameter) of 0.21 and 0.32 . The CG of 0.21 corresponds to an early estimate of the MSL CG location while the 0.32 value corresponds to an $L / D$ value of approximately 0.24 at a velocity of $4700 \mathrm{~m} / \mathrm{s}$ (Mach 24). In addition, the radial CG off set for this configuration was designed to be zero. For the determination of ballistic coefficient, a mass of 2200 $\mathrm{kg}$. was used.

At the axial CG of 0.21 , the trim angle of attack depicts oscillatory behavior of 1 degree within the Mach 2.3 to 4.5 Unitary experimental data range before reaching a maximum value of 15.4 degrees at a velocity of approximately $1300 \mathrm{~m} / \mathrm{s}$ (Mach 6). Beyond Mach 6, the trim angle of attack at the forward CG decreases nearly linear to a continuum limit value of approximately 12.8 degrees. Likewise, the $\mathrm{L} / \mathrm{D}$ values depict the same low supersonic behavior as well as the nearly linear behavior beyond the maximum L/D of 0.266 at Mach 4.5.

At the axial CG of 0.32 , the trim angle of attack depicts a larger variation in trim angle of attack of about 2.5 degrees within the Unitary data range and also reaching a maximum of 16.9 degrees at a velocity of approximately $550 \mathrm{~m} / \mathrm{s}$ (Mach 2.7) while reaching another lower peak of 16.1 degrees at Mach 6. Beyond Mach 6 , the trim angle of attack at the 0.32 CG decreases nearly linear to a continuum limit value of approximately 13.6 degrees. As with the forward CG results, the $\mathrm{L} / \mathrm{D}$ shows a corresponding maximum value at the peak trim reaching an overall maximum of 0.298.

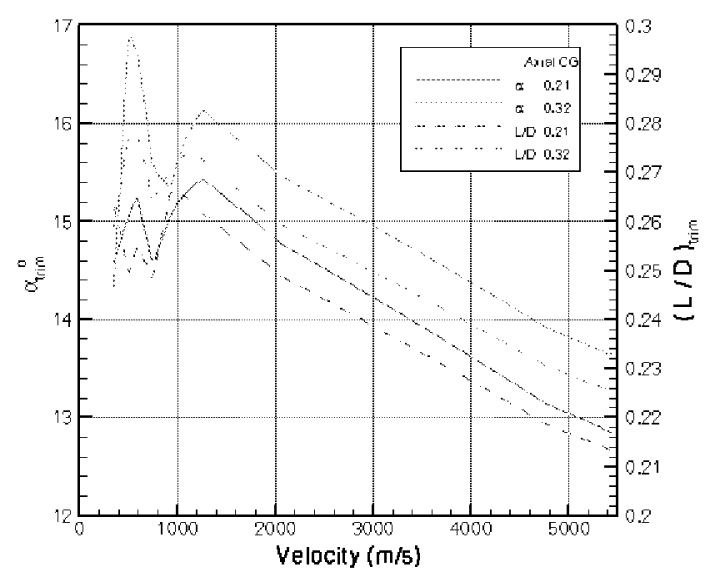

Figure 17. L/D Continuum Trim Characteristics for Shelf-All Configuration.

At trim conditions, the ballistic coefficient for the forward CG shows no low supersonic oscillatory behavior while approaching a maximum of 114 at Mach 6 while the axial CG of 0.32 showed the same distribution while displaying a maximum of 116 at Mach 6. As expected, these results show a low sensitivity to CG 
location due to only a small (1\%) difference in ballistic coefficient over the entire continuum regime.

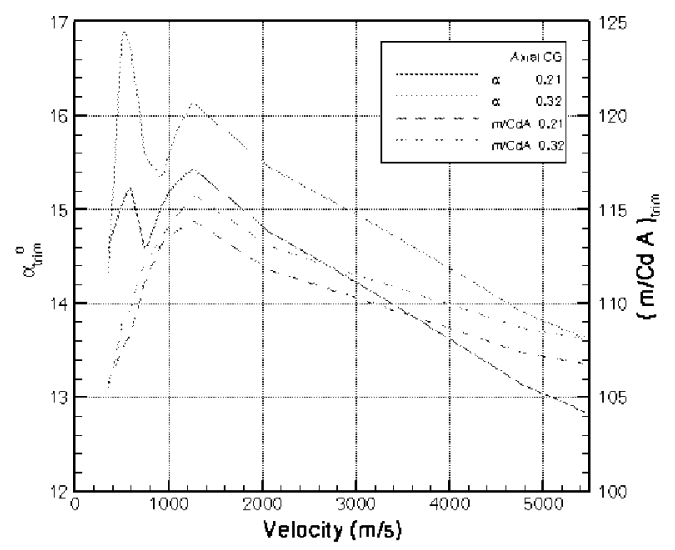

Figure 18. Ballistic Coefficient Continuum Trim Characteristics for Shelf-All Configuration.

\section{Flight Results-(Shelf-All Non-Continuum Database)}

For the rarefied regime, an existing set of free molecular data ${ }^{2}$ over an angle of attack range of -180 to 180 degrees was used in conjunction with bridging function ${ }^{20}$ to provide aerodynamic values within the transition atmospheric regime. Figures 19 and 20 show the trim characteristics for the Shelf-All configuration from the edge of continuum to a value of 20 . A Knudsen number $(\mathrm{Kn})$ of 20 was sufficient to cover the range up to the atmospheric interface at approximately 125000 meters above ground level.

Figure 19 shows the oscillatory behavior of the pitching moment within the transition regime. At the continuum limit and up to a Knudsen number of approximately 5 , there exists only a single trim point that is also stable (negative $\mathrm{Cm}_{\alpha}$ ). As the vehicle descends through the transition regime, there are multiple conditions that the vehicle could potentially trim, but could potentially be an unstable or stable trim point. Figure 20 displays Knudsen number and $\mathrm{Cm}$ alpha as a function of trim angle of attack $\left(\alpha_{\text {trim }}\right)$. For Knudsen numbers above 2 , there exist three trim angles of which 2 are unstable and 1 is stable. This information is needed while designing a guidance controller during the entry phase of the trajectory.

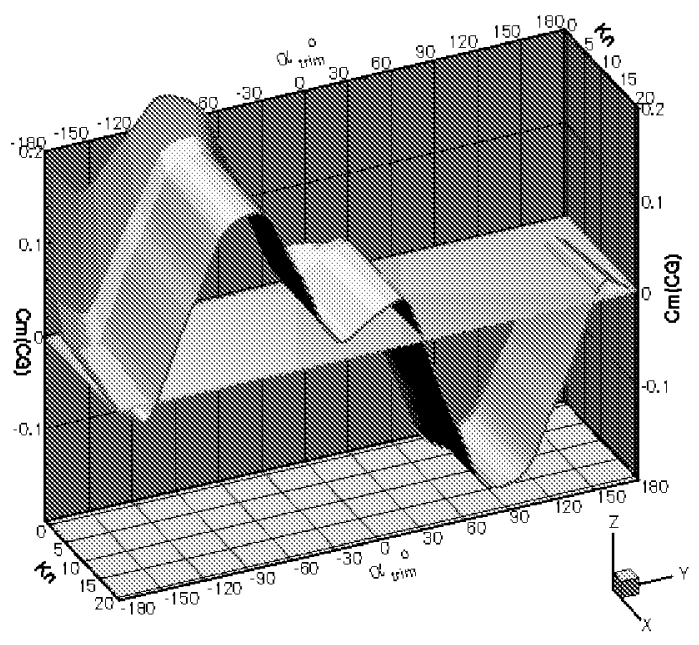

Figure 19. Non-Continuum Pitching Moment for Shelf- All Configuration.

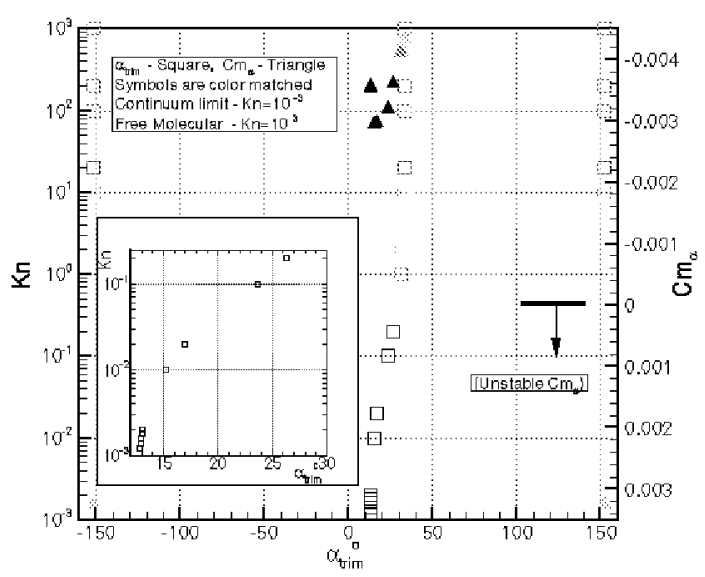

Figure 20. Non-Continuum Trim Characteristics for Shelf-All Configuration.

\section{Concluding Remarks}

The CFD validation of USM3Dns and LAURA with the Unitary experimental data, the use of intermediate LAURA check analyses, as well as the validation of FELISA with the Mach $6 \mathrm{CF}_{4}$ experimental data provided very good agreement and a higher confidence in each CFD code's aerodynamic analysis results including the longitudinal static trim characteristics for the Mars continuum atmosphere. 


\section{Acknowledgments}

The authors would like to thank Mr. Steve Alter of the NASA LaRC Aerothermodynamics Branch for creating the structured grids used in the LAURA analyses and providing the surface topology used for the USM3D unstructured grid generation. The authors would also like to thank Mr. Ed Parlette of Vigyan Inc. for creating the unstructured grids used in the USM3D analyses. In addition, thanks are due to Dr. Neil Cheatwood of the NASA LaRC Exploration Programs Office for his helpful recommendations and paper review.

\section{References}

1. Lockwood M.K., Powell R.W., Graves C.A. and Carman G.L. Entry System Design Considerations for Mars Landers, American Astronautical Society Paper No. 01-023, 24 ${ }^{\text {th }}$ AAS Guidance and Control Conference, January 31-February 4 2001, Breckenridge, $\mathrm{CO}$.

2. Striepe S.A., Queen E.M., Powell R.W., Braun R.D., Cheatwood F.M., Aguirre J.T., Sachi L.A. and Lyons D.T., An Atmospheric Guidance Algorithm Testbed for Mars Surveyor Program 2001 Orbiter and Lander, AIAA Paper 98-4569, Aug 1998.

3. Braun R.D., Powell R.W., Cheatwood F.M., Spencer D.A., and Mase R.A. The Mars Surveyor 2001 Lander: A First Step Toward Precision Landing, $49^{\text {th }}$ International Astronautical Congress, Melbourne, Australia, IAF-98-Q.3.03, Sept 28-Oct 21998 .

4. Bauer G.L., Cornick D.E., and Stevenson R., Capabilities and Applications of the Program to Optimize Simulated Trajectories (POST), NASA CR2770, February 1977.

5. Frink N.T., Tetrahedral Unstructured NavierStokes Method for Turbulent Flows, AIAA Journal, Vol. 36, No. 11, pp. 1975-1982, November 1998.

6. Roe P.L., Approximate Riemann Solvers, Parameter Vectors, and Difference Schemes, Journal of Computational Physics, Vol. 43, No. 2, pp. $357-$ 372, October 1981.

7. Bhat M. K. and Parikh P. C., Parallel Implementation of an Unstructured Grid-Based Navier-Stokes Solver, AIAA Paper 99-0663, January 1999.
8. Wada Yasuhiro and Liou Meng-Sing, An Accurate and Robust Flux Splitting Scheme for Shock and Contact Discontinuities, SIAM Journal of Scientific Computations, Vol. 18, No. 3, pp. 633-657, May 1997.

9. Gnoffo P.A., An Upwind-Biased, Point-Implicit Relaxation Algorithm for Viscous Compressible Perfect Gas Flows, NASA TP 2953, Feb. 1990.

10. Cheatwood F. M. and Gnoffo P. A., User's Manual for the Langley Aerothermodynamic Upwind Relaxation Algorithm (LAURA), NASA Technical Memorandum 4674, April 1996.

11. Riley, C.J. and Cheatwood, F.M., DistributedMemory Computing with the Langley Aerothermodynamic Upwind Relaxation Algorithm (LAURA), Advances in Engineering Software, Vol. 29, No. $3-$ 6, July 1998, pp. 317-324.

12. Yee H.C., On Symmetric and Upwind TVD Schemes, NASA TM 86842, 1955.

13. Peiro J., Peraire J., and Morgan K., FELISA System Reference Manual and Users Guide, NASA CP3291, May 1995.

14. Bibb K.L., Peraire J., and Riley C.J., Hypersonic Flow Computations On Unstructured Meshes, AIAA Paper 97-0625, January 1997.

15. Wilmoth R.G., LeBeau G.J., and Carlson A.B., DSMC Grid Methodologies for Computing Low Density Hypersonic Flows About Reusable Launch Vehicles, AIAA Paper 96-1812, June 1996.

16. Bird G.A., Molecular Gas Dynamics and Direct Simulation of Gas Flows, Clarendon Press, Oxford, 1994.

17. Murphy K.J., Horvath T.J., Erickson G.E., and Green J.M., Supersonic Aerodynamic Characteristics of Proposed Mars '07 Smart Lander Configurations, AIAA 2002-4409, August 2002.

18. Gnoffo P.A., Braun R.D., Weilmuenster K.J., Mitcheltree R.A., Engelund W.C., and Powell R.W., Prediction and Validation of Mars Pathfinder Hypersonic Aerodynamic Database, Journal of Spacecraft and Rockets, Vol. 36 No. 3, MayJune 1999. 
19. Horvath T.J., O'Connell T., Cheatwood F.M., and Alter S. Experimental Hypersonic Aerodynamic Characteristics of the Mars Surveyor 2001 Precision Lander with Deployable Flap, AIAA 20024408, August 2002.

20. Celenligil M.C., Moss J.N. and Blanchard R.C., Three-Dimensional Flow Simulation about the AFE Vehicle in the Transitional Regime, AIAA 890245, January 1989. 